\title{
Effect of Hemoperfusion Using Polymyxin B-Immobilized Fiber on IL-6, HMGB-1, and IFN Gamma in a Neonatal Sepsis Model
}

\author{
MOHAMED HAMED HUSSEIN, TAKENORI KATO, TAKAHIRO SUGIURA, GHADA A. DAOUD, \\ SATOSHI SUZUKI, SUMIO FUKUDA, HISANORI SOBAJIMA, INEKO KATO, AND \\ HAJIME TOGARI
}

Department of Pediatrics, Neonatology and Congenital Disorders [M.H.H., T.K., T.S., G.A.D., S.F., H.S., I.K., H.T.], Nagoya City University Graduate School of Medical Sciences, Nagoya, Japan, Department of Pediatrics [S.S.], Nagoya City Johoku Hospital, Nagoya, Japan, Neonatal Intensive Care Unit [M.H.H.], Pediatric Hospital, Cairo University, Cairo, Egypt, Maternal and Child Health Department

[M.H.H., G.A.D.], VACSERA, Giza, Egypt

\begin{abstract}
To evaluate effects of polymyxin B direct hemoperfusion (PMX-DHP) on a neonatal sepsis cecal ligation and perforation (CLP) model, in 24 anesthetized and mechanically ventilated 3 -d-old piglets, 16 were assigned to CLP and an arteriovenous extracorporeal circuit from $3 \mathrm{~h}$ until $6 \mathrm{~h}$ post-CLP, with a PMX-column in PMX-DHP-treated group (8 piglets) and 8 as sham. Plasma lipopolysaccharide (LPS) was measured at before CLP and at 3 and $9 \mathrm{~h}$. Changes in mean systemic blood pressure (mSBP), mean pulmonary blood pressure, serum IL-6, tumor necrosis factor alpha, interferon gamma, and highly mobile group-1 box protein were measured before CLP and at 1, 3, 6, and $9 \mathrm{~h}$. LPS was lower in the sham and PMX-DHP groups than in the control at $9 \mathrm{~h}$. The mSBP was higher in the sham and PMX-DHP groups than in the control at both $6 \mathrm{~h}$. IL-6 was lower in the sham and PMX-DHP groups than in the control at $6 \mathrm{~h}$. HMGB-1 was lower in the PMX-DHP group than in the control
\end{abstract}

ABSTRACT

at $6 \mathrm{~h}$. IFN- $\gamma$ was only detected in the control group at $9 \mathrm{~h}$. Survival times in the PMX-DHP group were longer than in the control. Thus, PMX-DHP improved septic shock in a neonatal septic model. (Pediatr Res 58: 309-314, 2005)
Abbreviations
CLP, cecal ligation and perforation
CVP, central venous pressure
HMGB-1, highly mobile group-1 box protein
IFN- $\boldsymbol{\gamma}$, interferon gamma
LPS, lipopolysaccharide (endotoxin)
mPAP, mean pulmonary blood pressure
mSBP, mean systemic blood pressure
PMX-DHP, polymyxin B direct hemoperfusion
TNF- $\alpha$, tumor necrosis factor alpha

A study in the United States estimated that severe sepsis accounts for $2-11 \%$ of all admissions to hospitals or intensive care units (1). The numbers become more striking when we focus on neonatal sepsis. According to a World Health Organization report in 1998, in developed countries, the incidence of neonatal sepsis is between 1 and 10 in 1000 in term infants and more frequent in preterms, and it is estimated that the incidence in developing countries is higher (2).

During serious Gram-negative bacterial infection, the release of LPS from the outer membrane of Gram-negative

Received June 21, 2004; accepted November 15, 2004

Correspondence: Mohamed Hamed Hussein, M.D., Department of Pediatrics, Neonatology and Congenital Disorders, Nagoya City University Graduate School of Medical Sciences, 1 Kawasumi, Mizuho-cho, Mizuho-ku, Nagoya 467-8601, Japan; e-mail: drmhamed@yahoo.com or hamed@med.nagoya-cu.ac.jp

Toray Industries (Tokyo, Japan) provided the polymyxin B immobilized fiber columns. DOI: 10.1203/01.PDR.0000169995.25333.F7 bacteria provokes excessive production and secretion of proinflammatory cytokines, a process that plays an important role in the development of sepsis syndrome (3).

IL-6 appears to be a promising candidate cytokine for diagnosis of neonatal septicemia (4), as it acts as a marker of the activation status of the cytokine network and reflects the influence of several other cytokines (5). Furthermore, higher concentrations portend a poor outcome (6).

On the other hand, IFN- $\gamma$ plays an important role in the pathophysiology of sepsis in CLP models and intra-abdominal sepsis, as it contributes to lung inflammation (7), and antiIFN- $\gamma$ antibodies seal the cecum and reduce bacterial movement into the peritoneum (8).

Also, HMGB-1, a protein previously known only as a nuclear transcription factor, is now implicated as a mediator of delayed endotoxin lethality and systemic inflammation (9). 
Polymyxin B, a well-known antibiotic, is a cyclic lipophilic peptide that inactivates endotoxins due to its high affinity for the lipid-A moiety substance in LPS (10). Thus, polymyxin B prevents the generation of superoxide production by macrophages in vitro (11). Furthermore, it prevented endotoxininduced mortality of mice (12) and rabbits (13). Applying PMX-DHP, where polymyxin-B is attached to polystyrene fiber with covalent bonding without its release, has shown to be effective in the clearance of LPS and yields a significant improvement of the survival rate in septic shock adult patients (14). Treating sepsis in neonates with PMX-DHP needs to be fully studied before its application in human neonatal sepsis. The host response to bacteria is different in the neonate than in the adult (15), and, therefore, the protective mechanisms of this therapy may be different or manifest themselves in another manner than that observed in adults. Thus, applying PMXDHP in a neonatal sepsis model is important before its clinical application.

We have designed a modified neonatal CLP sepsis model (16) that yields a blood culture of Gram-negative, anaerobes and Gram-positive bacteria. The model also showed changes seen in neonatal sepsis in the form of progressive decrease in mSBP with a significant increase in the ratio of the mPAP to the mSBP. There were significant elevations in plasma LPS, serum TNF- $\alpha$, and IL-6 levels. The model had a survival time of $10.4 \pm 1.3 \mathrm{~h}$ (mean \pm SEM).

This study is the first report on evaluating PMX-DHP effects in neonatal sepsis CLP model and the first to evaluate its effect on IFN- $\gamma$ and HMGB-1.

\section{MATERIALS AND METHODS}

Animal preparation. The experiments were performed in adherence with the National Institutes of Health guidelines on the use of experimental animals, and the protocol was approved by the Ethics Committee of Nagoya City University Graduate School of Medical Sciences. Twenty-four (eight in each group: PMX-DHP, control, and sham) mixed-strain newborn piglets were obtained on their third day of life from a local farmer $30 \mathrm{~min}$ from the laboratory. The piglets were transported on the day of the procedure. The mean weight was $1686.7 \mathrm{~g}$ (SEM, $41.8 \mathrm{~g}$ ) in PMX-DHP group, $1640 \mathrm{~g}$ (SEM, $62.7 \mathrm{~g}$ ) in the control group, and $1715 \mathrm{~g}$ (SEM, $47 \mathrm{~g}$ ) in the sham group. The piglets were premedicated with ketamine chloride $(10 \mathrm{mg} / \mathrm{kg}$ intramuscularly), after which anesthesia was induced with pentobarbital sodium $(20 \mathrm{mg} / \mathrm{kg}$ i.v. $)$ and was maintained by continuous infusion of pentobarbital sodium $(5 \mathrm{mg} / \mathrm{kg} / \mathrm{h})$ in $5 \%$ glucose solution via a peripheral line at a rate of $5 \mathrm{~mL} / \mathrm{kg} / \mathrm{h}$ throughout the study to avoid hypovolemia. All surgical procedures were performed under sterile conditions. Each piglet underwent a tracheotomy and was intubated with an endotracheal tube (internal diameter of $4.0 \mathrm{~mm}$ ) and ventilated with an infant ventilator (model IV-100, Sechrist Industries, Anaheim, CA). The inspiration/expiration pressures were initially set at $14 / 4 \mathrm{cmH}_{2} \mathrm{O}$, with an inspiration time of $0.5 \mathrm{~s}$ using room air. A cut-down procedure was used to insert a $3 \mathrm{~F}$ polyvinyl catheter into the left femoral artery for measuring the mSBP. A 4-Fr Berman angiographic catheter (American Edwards Laboratories, Irvine, CA) was inserted under fluoroscopy via the right external jugular vein and placed in the main pulmonary artery to measure the mPAP, and a $4 \mathrm{~F}$ polyvinyl catheter was inserted via the left external jugular vein into the right atrium to measure the CVP. The $\mathrm{mSBP}, \mathrm{mPAP}$, and CVP were measured with a neonatal monitor (model 78801 B, Hewlett Packard, Andover, MA). A MacLab/8s system (ADI Instruments, Mountain View, CA) was used for data collection. Body temperature was maintained by a thermal pad and monitored by a rectal probe. Modified cecal ligation and perforation (cecal devascularization and perforation) was performed on the PMX-DHP and control groups. Briefly, a para-median incision approximately $4-\mathrm{cm}$ long was made, sufficient to expose the cecum and terminal ileum. The ileocecal artery was identified and ligated near the cecum resulting in devascularization of the distal end of the cecum. A 1-cm incision was made in the antimesenteric side. The cecum was gently milked to extrude feces into the peritoneal cavity. Then the abdominal incision was closed in two layers.

The study was continued to the time of spontaneous death or for $24 \mathrm{~h}$. Animals surviving to $24 \mathrm{~h}$ were killed with a lethal dose of pentobarbital sodium.

PMX-DHP treatment. The polymyxin B-immobilized fiber was produced by immobilizing polymyxin $\mathrm{B}$ on polystyrene fiber ( $0.5 \%$ weight ratio) using covalent bonding without its release. The column for DHP is formed of polycarbonate tube containing $4.5 \times 45 \mathrm{~cm}$ adsorbent of polymyxin Bimmobilized fibers supplied by Toray Industries (Tokyo, Japan). The filling capacity of the column is $10 \mathrm{~mL}$.

DHP was performed through an extracorporeal circuit formed of the PMX column connected with circuit tube formed of polyvinylchloride (AP chamber set $15 \mathrm{~m}$, Niporo Corp., Tokyo, Japan) and a peristaltic pump (Iwaki PST-110, Asahi Techno Glass, Tokyo, Japan) between the right femoral artery and left external jugular vein (arteriovenous DHP). The extracorporeal circuit and column was filled with $20 \mathrm{~mL}$ of blood from a donor piglet to avoid the effect of hypovolemia. Cross-matching was done before the CLP procedure. Blood flow rate through this circuit was $3 \mathrm{~mL} / \mathrm{kg} / \mathrm{min}$ (high-volume hemoperfusion) (17). The PMX-DHP started $3 \mathrm{~h}$ after the CLP was performed for $3 \mathrm{~h}$ until the $6 \mathrm{~h}$ sampling time. Volume resuscitation was performed one time with blood from the extracorporeal circuit $(5 \mathrm{~mL} / \mathrm{kg}$ i.v. over $30 \mathrm{~min})$ immediately after the 6-h blood samples were collected to counteract and/or avoid the capillary leakage effect of the extracorporeal circuit. Low-molecular-weight heparin was applied as an anticoagulant just before starting the DHP circuit.

Control group. Animals were treated with the same procedure described, except that the PMX column was replaced with a polyvinylchloride tube with the same filling volume $(10 \mathrm{~mL})$.

Sham group. The sham piglets underwent the para-median incision, only without CLP procedure or applying extracorporeal circuits.

Experimental protocol. In each group, blood samples for TNF- $\alpha$, IL-6, IFN- $\gamma$, and HMGB-1 assays were aseptically collected from the femoral arterial catheter. Blood samples were taken before CLP and at 1,3,6, and $9 \mathrm{~h}$ after CLP. Plasma LPS was measured before CLP and at 3 and $9 \mathrm{~h}$ after CLP. Blood samples in the sham group were taken at same time interval. Each sample was placed into pyrogen-free sterilized tubes.

Measurements. Plasma LPS levels were measured using a commercial Limulus amebocyte lysate assay kit (Toray Industries). Serum TNF- $\alpha$ and IL-6 were measured using an immunoassay kit specific for porcine TNF- $\alpha$ and IL-6, respectively (Genzyme-Techne (R\&D Systems), Minneapolis, MN). Serum IFN- $\gamma$ was measured using an ELISA kit specific for porcine IFN- $\gamma$ (BioSource International, Camarillo, CA). Duplicate measurements were performed for each sample. The concentrations were calculated based on the obtained standard curves. HMGB-1 was measured using ELISA with a MAb that does not cross-react with HMGB-2, as described by Yamada et al. (18).

Bactericidal activity test. This test was carried out to demonstrate whether polymyxin B-immobilized fibers have a bactericidal activity on Gram-negative bacteria. The bacterium used was Pseudomonas aeruginosa IFU-1327. The culture medium was calf serum. The medium used for bacterial colony formation was Trypto-Soya Agar (Nissui, Tokyo, Japan). Forty grams of Trypto-Soya Agar contains $15 \mathrm{~g}$ of peptone, $5 \mathrm{~g}$ of soybean peptone, $5 \mathrm{~g}$ of $\mathrm{NaCl}$, and $15 \mathrm{~g}$ of Agar. Added to this was $0.5 \mathrm{~g}$ of immobilized polymyxin $\mathrm{B}$ on polystyrene fiber or $0.5 \mathrm{~g}$ of polystyrene fiber without polymyxin $\mathrm{B}$ to 15 $\mathrm{mL}$ calf serum containing about $10^{6}$ colony-forming unit $(\mathrm{CFU}) / \mathrm{mL}$ bacteria. The culture was incubated at $37^{\circ} \mathrm{C}$ for $2 \mathrm{~h}$ and compared with the same amount of calf serum containing about $10^{6} \mathrm{CFU} / \mathrm{mL}$ bacteria without adding any fibers. CFU were determined after the incubation for $30 \mathrm{~min}$ and 1 and $2 \mathrm{~h}$.

Statistical analysis. The means of the three groups (intergroup) at the same time point and in the same group (intragroup) at different time points were compared using ANOVA for repeated measures, followed by the Tukey post hoc test. If the data were not normally distributed, the Kruskal-Wallis test was used. When significance was detected Mann-Whitney test was used. Data are reported as mean \pm SEM. Probability values $<0.05$ were considered significant. Differences in survival between the two groups were calculated using the Kaplan-Meier test and were compared using Kruskal-Wallis test followed by Mann-Whitney test. All data analyses were performed with the commercially available statistical analysis software package SPSS (Chicago, IL).

\section{RESULTS}

Effect on endotoxin (LPS). Plasma LPS showed no differences between the three groups at before CLP time, but was higher in both PMX-DHP and control groups than in the sham group at 3 and $9 \mathrm{~h}$ (Table 1). Plasma LPS showed no differences between the PMX-DHP group and the control at $3 \mathrm{~h}$. At 
Table 1. Plasma endotoxin mean \pm SEM $\mathrm{pg} / \mathrm{ml}$

\begin{tabular}{lccc}
\hline \multicolumn{1}{c}{ Time } & Before CLP & $3 \mathrm{hr}$ & $9 \mathrm{hr}$ \\
\hline Sham & $27 \pm 2.18$ & $25.8 \pm 1.78$ & $21.5 \pm .889$ \\
& $(n=8)$ & $(n=8)$ & $(n=8)$ \\
Control & $39.9 \pm 10.4$ & $868.6 \pm 376.3 \dagger$ & $3670.3 \pm 1519 \S, \dagger$ \\
& $(n=8)$ & $(n=8)$ & $(n=7)$ \\
PMX-DHP & $53.8 \pm 20.8$ & $857.5 \pm 442.2 \dagger$ & $685.7 \pm 299 \dagger$ \\
& $(n=8)$ & $(n=8)$ & $n=8)$ \\
\hline
\end{tabular}

$\S p<0.05$ (comparing between PMX-DHP and Control).

$\dagger p<0.005$ (comparing to sham).

$9 \mathrm{~h}$, the plasma LPS in the PMX-DHP group was lower than in the control.

Effect on blood pressure. The mSBP in both the PMX-DHP and control groups decreased after $1 \mathrm{~h}$ and became lower when compared with before CLP in the PMX-DHP group only at $9 \mathrm{~h}$ $(62.4 \pm 2.2 \mathrm{~mm} \mathrm{Hg}$ versus $41.2 \pm 5.2 \mathrm{~mm} \mathrm{Hg}, p<0.01$, ANOVA), but in the control group at both 6 and $9 \mathrm{~h}(69.6 \pm$ $3 \mathrm{~mm} \mathrm{Hg}$ versus $32.6 \pm 4.5 \mathrm{~mm} \mathrm{Hg}$ and $25.6 \pm 4 \mathrm{~mm} \mathrm{Hg}$, respectively, $p<0.01$, ANOVA). The mSBP in the sham group showed no differences at different time points. There were no differences between the three groups in their $\mathrm{mSBP}$ at before CLP or at 1 and $3 \mathrm{~h}$. At $6 \mathrm{~h}$, the mSBP in the control group became lower than in both the PMX-DHP and sham groups $(32.6 \pm 4.5 \mathrm{~mm} \mathrm{Hg}$ versus $55.1 \pm 4.9 \mathrm{~mm} \mathrm{Hg}$ and $58.61 \pm 6.3 \mathrm{~mm} \mathrm{Hg}, p<0.005$ and $p<0.001$, respectively). At $9 \mathrm{~h}$, the mSBP in the control group remained lower than in the PMX-DHP and sham groups $(25.6 \pm 4 \mathrm{~mm} \mathrm{Hg}$ versus 41.2 $\pm 5.2 \mathrm{~mm} \mathrm{Hg}$ and $61.9 \pm 6.9 \mathrm{~mm} \mathrm{Hg}$, respectively, $p<0.05$ and $p<00.005)$. The mSBP in the PMX-DHP group was also lower than in the sham group at $9 \mathrm{~h}(p<0.05)$ (Fig. 1-1).

The mPAP in both the PMX-DHP and the control group became higher at $6 \mathrm{~h}$ when compared with before CLP mPAP
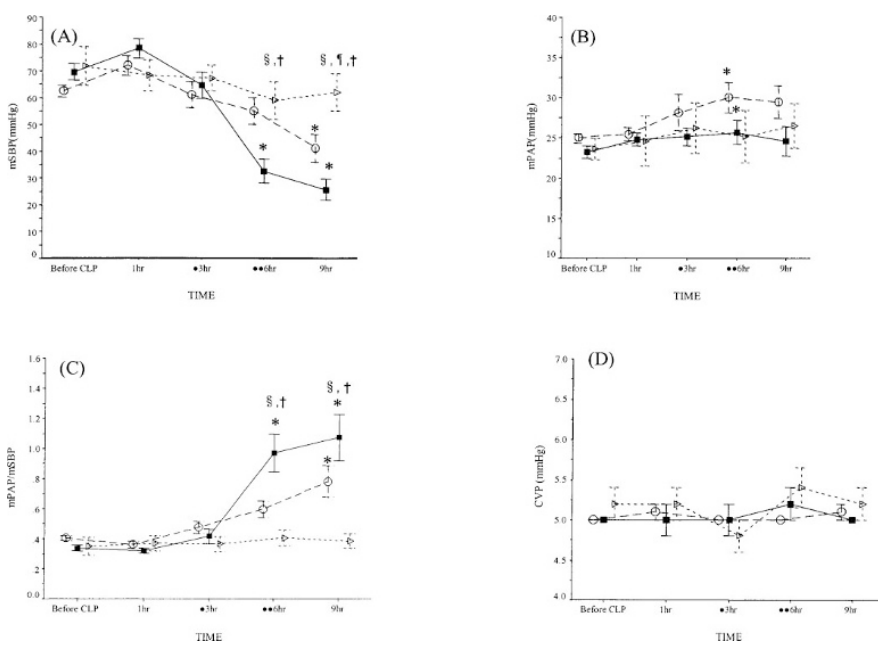

Figure 1. Serial blood pressure measurements in PMX-DHP-treated CLP neonatal sepsis model (circles; $n=8$ ), control (squares; $n=8$, except at $9 \mathrm{~h} n=7$ ), and sham groups (triangles; $n=8$ ): 1) $\mathrm{mSBP}, 2) \mathrm{mPAP}, 3) \mathrm{mPAP} / \mathrm{mSBP}$, and 4 ) CVP. $* 3 \mathrm{~h}$ is time of starting the extracorporeal circuit; $* * 6 \mathrm{~h}$ is time of stopping the extracorporeal circuit. §Difference between PMX-DHP and control groups $(\S p<0.05$ and $\S \S p<0.005)$. IDifference between PMX-DHP and sham groups ( $\mathbb{I} p<0.05$ and $\mathbb{\Phi} \llbracket \mid p<0.005$ ). $\dagger$ Difference between control and sham groups $(\dagger p<0.05$ and $\dagger \dagger p<0.005)$. * Intragroup significant difference compared before CLP time measurement. $\S, I, \dagger, *$ ANOVA for repeated measures followed by Tukey test as post hoc. Error bar represents SEM. in the PMX-DHP group ( $31.2 \pm 2 \mathrm{~mm} \mathrm{Hg}$ versus $25 \pm 0.6 \mathrm{~mm}$ $\mathrm{Hg}, p<0.01$, ANOVA) and in the control group $(27.3 \pm 2.1$ $\mathrm{mm} \mathrm{Hg}$ versus $23.2 \pm 0.6 \mathrm{~mm} \mathrm{Hg}, p<0.01$, ANOVA). At $9 \mathrm{~h}$, the mPAP were still higher than before CLP, but the differences did not reach significance. The mPAP in the sham group showed no differences at different time points and there were no significant intergroup differences in mPAP (Fig. 1-2).

The $\mathrm{mPAP} / \mathrm{mSBP}$ ratio became higher in both the PMXDHP and the control group when compared with before CLP at $9 \mathrm{~h}$ in the PMX-DHP group $(0.4 \pm 0.02$ versus $0.8 \pm 0.1, p<$ 0.01, ANOVA) and at 6 and $9 \mathrm{~h}$ in the control group $(0.3 \pm .02$ versus $1 \pm 0.1$ and $1.1 \pm 0.1$, respectively, $p<0.01$, ANOVA). The $\mathrm{mPAP} / \mathrm{mSBP}$ ratio in the sham group showed no differences at different time points. The $\mathrm{mPAP} / \mathrm{mSBP}$ ratio was higher in the control group than in both the PMX-DHP and sham groups at $6 \mathrm{~h}(1 \pm 0.1$ versus $0.6 \pm 0.1$ and $0.41 \pm 0.05$, $p<0.05$ and $p<0.01$, respectively) and at $9 \mathrm{~h}$ time $(1.1 \pm 0.1$ versus. $0.8 \pm 0.1$ and $0.380 \pm 0.05, p<0.05$ and $p<0.005$, respectively) (Fig. 1-3).

The main CVP showed no changes throughout the study in the three groups (Fig. 1-4).

Effect on body temperature. Similar changes accrued in the body temperature in the PMX-DHP and the control groups. Thus, we compared the sum of the two groups' temperatures to the sham group's temperatures. It increased from before CLP to the 1-h reading $\left(37.9 \pm 0.3^{\circ} \mathrm{C}\right.$ versus $39.2 \pm 0.4^{\circ} \mathrm{C}, p<$ 0.05 , ANOVA), and from the 1 -h reading to the 3 -h reading $\left(41 \pm 0.4^{\circ} \mathrm{C}, p<0.05\right.$, ANOVA). However, readings at $6 \mathrm{~h}$ $\left(38.6 \pm 0.2^{\circ} \mathrm{C}\right)$ were lower in comparison with 3 -h readings, $(p$ $<0.05$, ANOVA), due to the effect of extracorporeal circula-
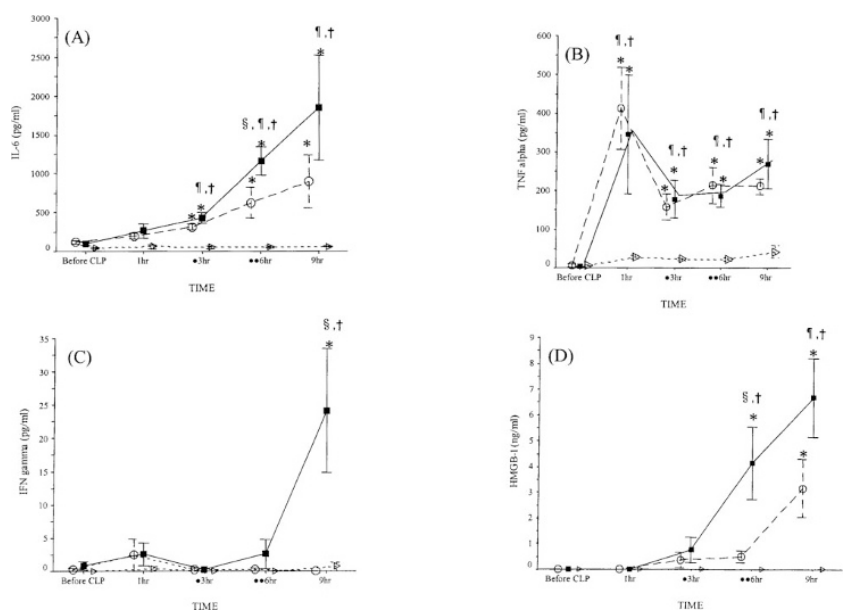

Figure 2. Serial serum levels of mediators in PMX-DHP-treated CLP neonatal sepsis model (circles; $n=8$ ), control (squares; $n=8$, except at $9 \mathrm{~h} n=$ 7), and sham groups (triangles; $n=8$ ): 1) IL-6, 2) TNF- $\alpha$, 3) IFN- $\gamma$, and 4) HMGB-1. $* 3 \mathrm{~h}$ is time of starting the extracorporeal circuit; $* * 6 \mathrm{~h}$ is time of stopping the extracorporeal circuit. §Difference between PMX-DHP and control groups $(\S p<0.05$ and $\S \S p<0.005)$. IDifference between PMX-

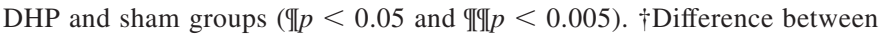
control and sham groups $(\dagger p<0.05$ and $\dagger \dagger p<0.005)$. * Intragroup significant difference compared with before CLP time measurement ${ }^{*} p<$ 0.01 , ANOVA for repeated measures, followed by the Tukey post hoc test. $\S, I, \dagger$ ANOVA for repeated measures, followed by the Tukey post hoc test. If the data were not normally distributed, the Kruskal-Wallis test was used; when significances were detected, Mann-Whitney test was used. Error bar represents SEM. 
tion in both groups. At $9 \mathrm{~h}$, readings returned again to near 3-h readings $\left(40.7 \pm 0.4^{\circ} \mathrm{C}\right)$. At $3 \mathrm{~h}$, the temperatures were higher in both the PMX-DHP and the control groups than in the sham group $\left(41 \pm 0.4^{\circ} \mathrm{C}\right.$ versus $\left.39 \pm 0.4^{\circ} \mathrm{C}, p<0.01\right)$. Temperature in the sham group showed no differences at different time points.

Effect on mediators. Serum levels of TNF- $\alpha$, IL-6, and IFN- $\gamma$ showed no differences between the three groups at before CLP, and both the PMX-DHP and the control groups continued to show no differences at 1 and $3 \mathrm{~h}$. Serum IL-6 levels in the PMX-DHP and control groups became higher than in the sham group at $3 \mathrm{~h}(312.8 \pm 39.4 \mathrm{pg} / \mathrm{mL}$ and $429.2 \pm$ $70.2 \mathrm{pg} / \mathrm{mL}$ versus $43.3 \pm 12 \mathrm{pg} / \mathrm{mL}, p<0.001)$, and remained higher at 6 and $9 \mathrm{~h}(6 \mathrm{~h}: 593.1 \pm 167.6 \mathrm{pg} / \mathrm{mL}$ and $1165.7 \pm 181.3 \mathrm{pg} / \mathrm{mL}$ versus $45.6 \pm 11.3 \mathrm{pg} / \mathrm{mL}, p<0.001$; 9 h: $693.9 \pm 403.1$ and $1847.1 \pm 677.7 \mathrm{pg} / \mathrm{mL}$ versus $51.8 \pm$ $11 \mathrm{pg} / \mathrm{mL}, p<0.001$, respectively). Serum levels of IL-6 in the PMX-DHP group were lower than in the controls at 6 and $9 \mathrm{~h}$, however, significance was only detected at $6 \mathrm{~h}(p<0.01)$ (Fig. 2-1).

Although, serum levels of TNF- $\alpha$ continued to show no differences between the control and PMX-DHP groups at 6 and $9 \mathrm{~h}$, serum levels of TNF- $\alpha$ in both groups were higher than in the sham group at 1, 3, 6, and $9 \mathrm{~h}, p<0.001$ (Fig. 2-2).

Serum IFN- $\gamma$ levels in the PMX-DHP group became lower after PMX-DHP therapy than in the control group, and this difference became significant at $9 \mathrm{~h}$ as IFN- $\gamma$ in the PMX-DHP group became undetectable and reached its peak in the control group $(24.1 \pm 9.3 \mathrm{pg} / \mathrm{mL}, p<0.05)$. Serum IFN- $\gamma$ in the sham group was undetectable throughout the study (Fig. 2-3).

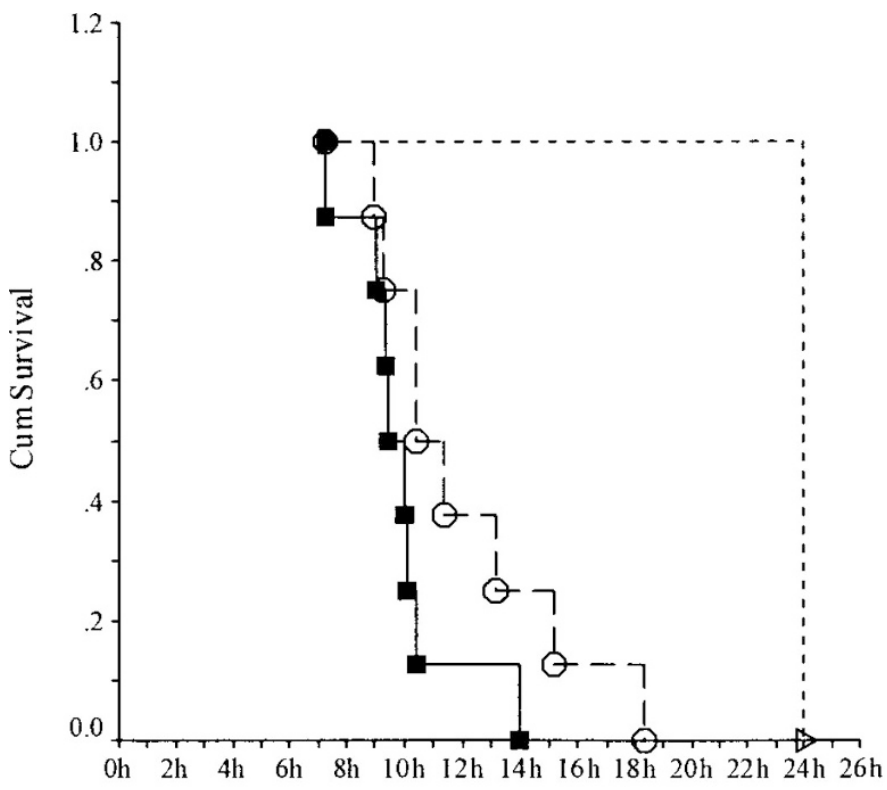

SURVI VAL TIME

Figure 3. Survival differences in PMX-DHP-treated (open circles) CLP neonatal sepsis model, control (black squares), and sham (open triangles) groups using Kaplan-Meier test. The survival times of PMX-DHP group were longer than in the control $(12.2 \pm 1.1 \mathrm{~h} v s 9 \pm .7 \mathrm{~h}, p<0.001$, Mann-Whitney test). In the PMX-DHP group 75\% reached the survival target (survival time in the control plus double its SEM) but only $12.5 \%$ in the control group. $0 \mathrm{~h}$ represents end of the CLP operation time.
Serum HMGB-1 became detectable in the serum samples of the PMX-DHP and control groups at $3 \mathrm{~h}$ and there were no differences between groups at that time. HMGB-1 was not detected in the sham group throughout the study. At $6 \mathrm{~h}$, serum HMGB-1 levels were higher in the control group than in the PMX-DHP group $(4.1 \pm 1.4 \mathrm{pg} / \mathrm{mL}$ versus. $0.48 \pm 0.2 \mathrm{pg} / \mathrm{mL}$, $p<0.05)$, and also higher than in the sham group $(p<0.001)$. At $9 \mathrm{~h}$, serum HMGB-1 levels were still higher in the control group but were only significant when compared with in the sham group $(p<0.0001)$. Serum HMGB-1 levels in the PMX-DHP group were higher than in the sham group only at 9 h $(p<0.05)$ (Fig. 2-4).

Effect on survival. The survival times of the PMX-DHP group were significantly longer than in the control group (12.2 $\pm 1.1 \mathrm{~h}$ versus $9 \pm 0.7 \mathrm{~h}, p<0.001$ ) (Fig. 3). The successful survival target is the survival time in the control group plus double its SEM. In the PMX-DHP group, 75\% reached the survival target, however, in the control group, only $12.5 \%$ did (Fig. 3). The entire sham group lived for $24 \mathrm{~h}$.

Effect on bacterial CFU (bactericidal activity test). There was no observed reduction of the CFU after incubation with immobilized polymyxin B on polystyrene fiber or with polystyrene fiber without polymyxin B (Fig. 4).

\section{DISCUSSION}

Targeting the blood LPS could be an ideal therapy for sepsis, especially lipid-A of the LPS (19), as considerable data have

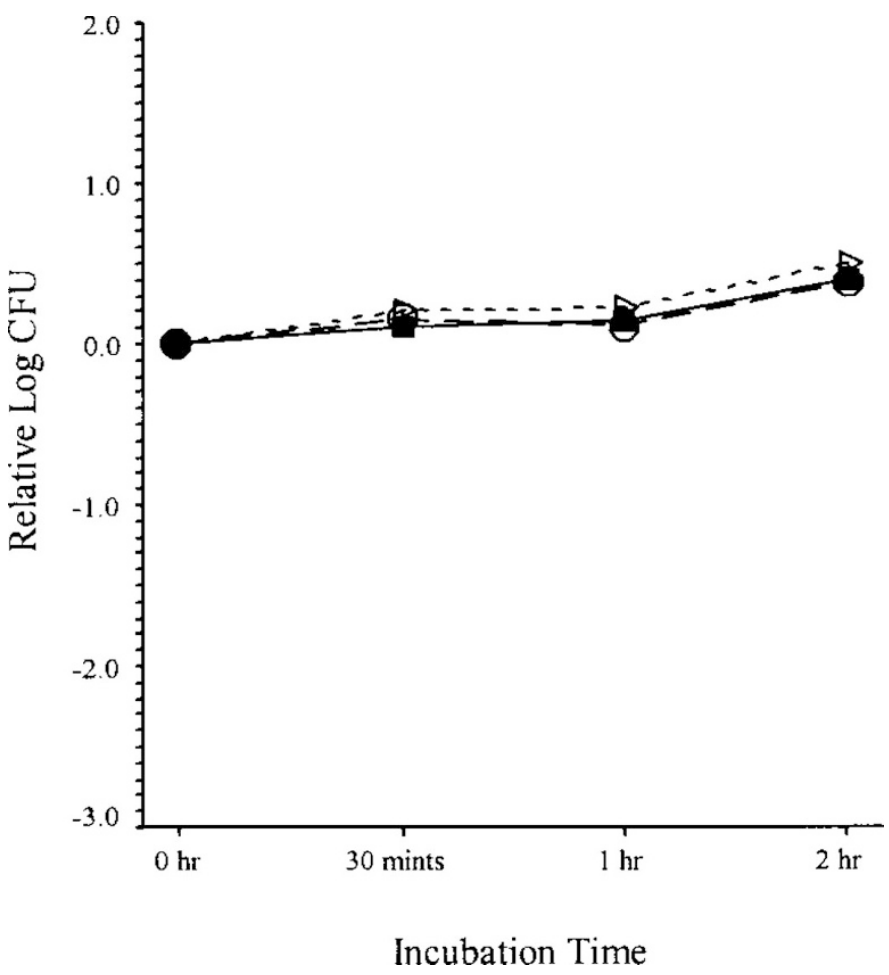

Figure 4. Bactericidal activity test. Comparison of the CFU of Pseudomonas aeruginosa IFU-1327 at different time points after i) adding $0.5 \mathrm{~g}$ of immobilized polymyxin $\mathrm{B}$ on polystyrene fiber open circles, black squares, open triangles (circles; $n=1$ ), ii) adding $0.5 \mathrm{~g}$ of polystyrene fiber without polymyxin $\mathrm{B}$ (squares; $n=1$ ), and iii) no adding of any fibers (triangles; $n=$ 1) to $15 \mathrm{~mL}$ calf serum containing about $10^{6} \mathrm{CFU} / \mathrm{mL}$ bacteria and incubated at $37^{\circ} \mathrm{C}$ for $2 \mathrm{~h}$. 
demonstrated that lipid-A is the toxic moiety of LPS, provoking cytokine release and pathophysiological effects of sepsis after administration (20). Furthermore, the lipid-A region of LPS is similar immunologically and biologically among most species of Gram-negative bacteria. Thus, reagents capable of neutralizing the lipid-A moiety of LPS would provide protective capacity against many different species of Gram-negative pathogens (21).

Clinical trials were conducted to examine the effect of anti-lipid-A murine MAb, but they did not provide evidence of survival benefit, probably because these reagents were not potent LPS antagonists (22).

The strong affinity of polymyxin B for lipid-A moiety substance of endotoxin makes it a suitable therapy (10), but it is toxic to both brain and kidney, which limits its parenteral use. On the other hand, using polymyxin B through an extracorporeal circuit in PMX-DHP was safe and also effective in adult septic shock patients (14).

In the recent study, applying PMX-DHP to a neonatal sepsis model also reduced the plasma LPS, (where it was five times higher in the control), regardless of the fact that the immobilized polymyxin B, as shown by the bactericidal activity test, lacks bactericidal activity.

The effect of PMX-DHP on the systemic blood pressure in sepsis models was the main concern of many studies. Yamamoto et al. (23) applied PMX-DHP in anesthetized adult sheep at the time of induction of sepsis by infusing $10 \mu \mathrm{g} / \mathrm{kg}$ of Escherichia coli-derived endotoxin. They concluded that PMX-DHP significantly improved the systemic hypotension caused by endotoxemia but had no effect on the pulmonary hypertension that developed in that model. Hanasawa et al. (24) showed a similar effect on systemic blood pressure in adult dogs that were intravenously treated with $1-5 \times 10^{9} \mathrm{E}$. coli/kg and, after $30 \mathrm{~min}$ from induction of sepsis the PMXDHP therapy was applied, but the differences in the mSBP between the treated and untreated groups were not significant in that study. However, in our study on a neonatal sepsis model, PMX-DHP therapy improved the systemic blood pressure and delayed the hypotensive effect of septic shock, although PMX-DHP showed no effect on the pulmonary hypertension that developed at $6 \mathrm{~h}$ in our CLP neonatal sepsis model, it lowered the $\mathrm{mPBP} / \mathrm{mSBP}$ ratio. This difference can be explained by the difference in the septic model used in each study.

Wang et al. (9) reported that serum HMGB-1 was detected late (after $8 \mathrm{~h}$ ) when injecting a median lethal dose (LD50) of LPS in mice and also that passive immunization using antiHMGB-1 improved the survival rates in those mice. Furthermore, in human serum, HMGB-1 was not detected in normal individuals but was detected in all critically ill patients with surgical sepsis and sepsis-induced organ dysfunction. Moreover, serum HMGB-1 levels were significantly higher in septic patients who did not survive when compared with survivors.

HMGB-1 in the neonatal CLP model was also detected as a late mediator, in comparison with $\mathrm{TNF}-\alpha$, but earlier than in the previous study (9), as it became detectable after $3 \mathrm{~h}$ from induction of sepsis, which is probably due to the severity of the insult.
Both Nakamura et al. (25) and Hanasawa et al. (26) concluded that the use of PMX-DHP in adult sepsis patients lowered the plasma concentration of IL- 6 both in survivors and nonsurvivors. Furthermore, Hanasawa and colleagues added that PMX-DHP decreased the serum levels of TNF- $\alpha$ in surviving sepsis patients but not in nonsurvivors.

The application of PMX-DHP in a neonatal sepsis model in our study caused a significantly lower level of IL-6, HMGB-1, and IFN- $\gamma$, however, there were no effects on TNF- $\alpha$. This might be because the application of PMX-DHP was after the TNF- $\alpha$ peak. An earlier application before the TNF- $\alpha$ peak at $1 \mathrm{~h}$ would be an early therapy before the marked elevation of other inflammatory mediators and the hypotensive effect caused by the LPS released from the CLP.

The use of a CLP model with a continuous release of fecal content to the abdominal cavity that acts as a septic focus makes it an ideal model to evaluate the LPS clearance by PMX-DHP and its effect on blood pressure changes and inflammatory mediators in neonatal sepsis. According to the classification of animal models of sepsis by Freise et al. (27), the CLP model is a septic model with an infectious focus. Freise and colleagues concluded that animal sepsis models that have a septic focus are closer to clinical reality where the infection spreads from a local focus to generalized septic shock. In addition, focal lesions, such as those observed in necrotizing enterocolitis, often lead to systemic sepsis state (28). Also, Goldfarb et al. (29) concluded that a porcine sepsis model shows very similar cardiovascular impairments to those noted in human sepsis. Furthermore, the PMX-DHP therapy was applied after $3 \mathrm{~h}$ from induction of sepsis, after elevation of plasma LPS and serum mediator levels, and after a decrease in the $\mathrm{mSBP}$ in comparison to before-CLP levels. Finally, our experiment showed the application of PMX-DHP on a neonatal septic model (not an adult one) using a specially made column with a lower filling volume. This is important because term and preterm neonatal immune systems show reduced host defense status to infection, which results from multiple factors, including hyporesponsiveness of the B cells and T cells (15), reduced quantities of granulocytes and their progenitors (30), and lower levels of cytokines (e.g. IL-6 production from monocytes and macrophages in response to IL-1 stimulation) (31).

We did not administer antibiotics to the animals in this study because, although antibiotics constitute the main therapy for Gram-negative bacterial infection and sepsis, they lack LPSneutralizing capacity (32). In addition, the administration of cell wall lytic antibiotics such as $\beta$-lactam drugs promotes the release of LPS in Gram-negative bacterial sepsis with unfavorable effects on the host (33).

The effect of PMX-DHP on serum IL-6, IFN- $\gamma$, and HMGB-1 suggests that it decreased the rate of production and/or release of these mediators, which is due to neutralizing the effect of LPS on the macrophages and monocytes by the polymyxin-B. This, in turn, caused improvement of the systemic blood pressure and resulted overall in a significant prolongation of the survival time and survival rate, as $75 \%$ of the PMX-DHP treated group reached the survival target in comparison with only $12.5 \%$ in the control group. However, a complete survival to $24 \mathrm{~h}$ time was not achieved, which is 
probably because we did not treat the focus of infection, which was the perforated cecum of the CLP model.

In summary, in a progressive neonatal sepsis CLP model, PMX-DHP therapy significantly lowered the plasma LPS levels, which improved the mSBP and significantly lowered serum levels of IL-6, HMGB-1, and IFN- $\gamma$, thereby causing improvement of survival time and rate. Thus, PMX-DHP improved the pathophysiologic events of septic shock in a CLP neonatal sepsis model and is worthy of further study for application in therapy of neonatal sepsis.

\section{REFERENCES}

1. Angus DC, Linde-Zwirble WT, Lidicker J, Clermont G, Carcillo J, Pinsky MR 2001 Epidemiology of severe sepsis in the United States: analysis of incidence, outcome, and associated costs of care. Crit Care Med 29:1303-1310

2. Postpartum care of mother and newborn: a practical guide. WHO/RHT/MSM/98.3

3. Hack CE, Aarden LA, Thijs LG 1997 Role of cytokines in sepsis. Adv Immunol 66:101-195

4. Silveira RC, Procianoy RS 1999 Evaluation of interleukin-6, tumor necrosis factoralpha and interleukin-1beta for early diagnosis of neonatal sepsis. Acta Paediatr 88:647-650

5. Casey LC, Balk RA, Bone RC 1993 Plasma cytokine and endotoxin levels correlate with survival in patients with the sepsis syndrome. Ann Intern Med 119:771-778

6. Sullivan JS, Kilpatrick L, Costarino AT Jr, Lee SC, Harris MC 1992 Correlation of plasma cytokine elevations with mortality rate in children with sepsis. J Pediat 120:510-515

7. Yin K, Hock CE, Lai PS, Ross JT, Yue G 1999 Role of interferon- $\gamma$ in lung inflammation following cecal ligation and puncture in rats. Shock 12:215-221

8. Qiu G, Wang C, Smith R, Harrison K, Yin K 2001 Role of IFN- $\gamma$ in bacterial containment in a model of intra-abdominal sepsis. Shock 16:425-429

9. Wang H, Bloom O, Zhang M, Vishnubhakat JM, Ombrellino M, Che J, Frazier A, Yang H, Ivanova S, Borovikova L, Manogue KR, Faist E, Abraham E, Andersson J, Andersson U, Molina PE, Abumrad NN, Sama A, Tracey KJ 1999 HMG-1 as a late mediator of endotoxin lethality in mice. Science 285:248-251

10. Malchesky PS, Zborowski M, Hou KC 1995 Extracorporeal techniques of endotoxin removal: a review of the art and science. Adv Renal Replac Ther 2:60-69

11. Takada H, Ogawa T, Yoshimura F, Otsuka K, Kokeguchi S, Kato K, Umemoto T, Kotani S 1988 Immunobiological activites of a porin fraction isolated from Fusobacterium nucleatum ATCC 10953. Infect Immun 56:855-863

12. Rifkind D 1967 Prevention by polymyxin B of endotoxin lethality in mice. J. Bacteriol 93:1463-1464

13. Corrigan JJ Jr., Kiernat JF 1979 Effect of polymyxin B sulfate on endotoxin activity in a gram-negative septicemia model. Pediatr Res 13:48-51

14. Tani T, Hanasawa K, Endo Y, Yoshioka T, Kodama M, Kaneko M, Uchiyama Y, Akizawa T, Takahasi K, Sugai K 1998 Therapeutic apheresis for septic patients with organ dysfunction: hemoperfusion using a polymyxin B immobilized column. Artif Organs 22:1038-1044

15. Andrew SK, Dianne EC 1996 The neonatal immune system. Semin Neonatal 1:67-75

16. Kato T, Hussein MH, Sugiura T, Suzuki S, Fukuda S, Tanaka T, Kato I, Togari H 2004 Development and characterization of a novel porcine model of neonatal sepsis. Shock 21:329-335

17. Grootendorst AF, van Bommel EF, van Leengoed LA, Nabuurs M, Bouman CS, Groeneveld AB 1994 High volume hemofiltration improves hemodynamics and survival of pigs exposed to gut ischemia and reperfusion. Shock 2:72-78

18. Yamada S, Inoue K, Yakabe K, Imaizumi H, Maruyama I 2003 High mobility group protein 1 (HMGB-1) quantified by ELISA with monoclonal antibody that does not cross-react with HMGB2. Clin Chem 49:1535-1537

19. Ray BL, Painter G, Raetz CR 1984 The biosynthesis of gram-negative endotoxin. Formation of lipid A disaccharides from monosaccharide precursors in extracts of Escherichia coli. J Biol Chem 259:4852-4859

20. Freudenberg M, Galanos C 1990 Bacterial lipopolysaccharides: structure, metabolism, and mechanisms of action. Int Rev Immunol 6:207-221

21. Elsbach P, Weiss J 1995 Prospects for use of recombinant BPI in the treatment of gram-negative bacterial infections. Infect Agents Dis 4:102-109

22. Bone RC, Balk RA, Fein AM, Perl TM, Wenzel RP, Reines HD, Quenzer RW, Iberti TJ, Macintyre N, Schein RM 1995 A second large controlled clinical study of E5, a monoclonal antibody to endotoxin: results of a prospective, multicenter, randomized, controlled trial. The E5 Sepsis Study Group. Crit Care Med 23:994-1005

23. Yamamoto H, Koizumi T, Kaneki T, Fujimoto K, Kubo K, Honda T 2002 Direct hemoperfusion with polymyxin B-immobilized fiber improves shock and hypoxemia during endotoxemia in anesthetized sheep. J Endotoxin Res 8:419-426

24. Hanasawa K, Tani T, Kodama M 1989 New approach to endotoxic and septic shock by means of polymyxin B immobilized fiber. Surg Gynecol Obstet 168:323-331

25. Nakamura T, Ebihara I, Shimada N, Koide H 1998 Changes in plasma erythropoietin and interleukin- 6 concentrations in patients with septic shock after hemoperfusion with polymyxin B-immobilized fiber. Intensive Care Med 24:1272-1276

26. Hanasawa K 2002 Extracorporeal treatment for septic patients: new adsorption technologies and their clinical application. Ther Apher 6:290-295

27. Freise H, Bruckner UB, Spiegel HU 2001 Animal models of sepsis. J Invest Surg 14:195-212

28. Edelson MB, Bagwell CE, Rozycki HJ 1999 Circulating pro- and counter inflammatory cytokine levels and severity in necrotizing enterocolitis. Pediatrics 103:766-771

29. Goldfarb RD, Glock D, Kumar A, McCarthy RJ, Mei J, Guynn T, Matushek M, Trenholme G, Parillo JE 1996 A porcine model of peritonitis and bacteremia simulates human septic shock. Shock 6:442-451

30. Cates KL, Rowe JC, Ballow M 1983 The premature infant as a compromised host. Curr Probl Pediatr 13:1-63

31. Schibler KR, Liechty KW, White WL, Rothstein G, Christensen RD 1992 Defective production of interleukin- 6 by monocytes: a possible mechanism underlying several host defense deficiencies of neonates. Pediatr Res 31:18-21

32. Tomasz A 1986 Penicillin-binding protein and the antibacterial effectiveness of beta-lactam antibiotics. Rev Infect Dis 8:S260-S278

33. Morrison DC, Bucklin SE 1996 Evidence for antibiotic-mediated endotoxin release as a contributing factor to lethality in experimental gram-negative sepsis. Scand J Infect Dis Suppl 101:3-8 